\title{
Accelerating diagnosis for childhood brain tumours: an analysis of the HeadSmart UK population data
}

Dhurgshaarna Shanmugavadivel, ${ }^{1}$ Jo-Fen Liu, ${ }^{1}$ Laura Murphy, ${ }^{1}$ Sophie Wilne,${ }^{2}$ David Walker, ${ }^{1}$ on behalf of the HeadSmart

${ }^{1}$ Children's Brain Tumour Research Centre, University of Nottingham, Nottingham, UK

2Paediatric Oncology, Nottingham University Hospitals NHS Trust, Nottingham, UK

Correspondence to: Dr Dhurgshaarna Shanmugavadivel, University of Nottingham

Children's Brain Tumour Research Centre, Nottingham NG7 2UH, UK;

\begin{abstract}
Background HeadSmart, a public and professional awareness campaign, was launched to enhance awareness of brain tumour symptomatology identified in the Royal College of Paediatrics and Child Health, National Institute for Health and Care Excellence- accredited guideline. Quality improvement data showed a reduction in diagnostic interval nationally. To reach the government target of 4 weeks, we need to identify subgroups with ongoing delays.
\end{abstract}

Methods Incident cases of brain tumours (0-18) diagnosed between January 2011 and May 2013 across 18 UK centres were included. Anonymised data including demographics, diagnosis and date of symptom onset/ presentation were collected. Key outcome measures, total diagnostic interval (TDI), patient interval (PI) and system interval (SI) were calculated. Subanalysis by age, tumour grade and location was also performed.

Results Young children (0-5 years) accounted for $38 \%$ of cases, with a peak age at diagnosis of 2 years. Central tumours experienced longest intervals with a median TDI of 10.5 weeks, PI of 3.2 weeks and SI of 2.9 weeks. Craniopharyngioma, low-grade glioma and optic pathway gliomas had the longest TDIs with a median of 15.1, 11.9 and 10.4 weeks, respectively. The greatest proportion of delay was in the SI. The 12-18 age group had a median TDI of 12.1 weeks, compared with 8 weeks for the 5-11 age group and 6 weeks for the

$0-5$ age group $(p<0.001)$.

Conclusions Clear patterns of intervals for different age groups and anatomical locations have been demonstrated. Tailoring education and awareness strategies to ensure earlier diagnosis for central tumours and young people is crucial to minimise brain injury, subsequent disability and late effects of treatment for $70 \%$ of survivors. 


\section{Introduction}

In the UK 450 children under the age of 16 are diagnosed with a brain tumour each year. ${ }^{1}$ The 5 year survival rate is more than $70 \%$ however two-thirds of survivors have a mild or moderate disability. $\stackrel{-}{ }$ Childhood brain tumours are the leading cause of cancer death but they pose a diagnostic challenge for primary and secondary care; the signs and symptoms that precede diagnosis are non-specific, fluctuate in severity and can mimic other common illnesses. A systematic review in 2005 identified that the total diagnostic interval (TDI), defined as the time from symptom onset to diagnosis, for childhood brain tumours in the UK ranked poorly to international comparisons. As a result, a high-quality evidence-based guideline was developed and published by the Royal College of Paediatrics and Child Health ( $\mathrm{RCPCH}$ ) in 2008 and subsequently disseminated in 2011 through a public and professional awareness campaign entitled "HeadSmart: Be brain tumour aware" to raise awareness of the symptoms and the need for timely imaging. ${ }^{34}$

This was associated with a reduction of the median total diagnostic interval from 14.4 weeks in 2006 to 6.7 weeks in 2013 ( $p<0.001$ ). ${ }^{-}$The publication of the UK Cancer Taskforce Strategy in 2015 stated all cancers should be diagnosed within 4 weeks by 2020 and has highlighted HeadSmart for evaluation. ${ }_{-}^{6}$ To further reduce the TDI for brain tumours in children in the UK, subgroups with the greatest risk of delay need to be identified in order to provide specifically tailored materials for health- care professionals and the public. Presentation of brain tumours is both age and location dependent. Previous studies have identified that those with shorter TDIs have more aggressive tumours, that is, there is an inverse relationship between TDI and survival. - High-grade gliomas commonly have rapid onset of symptomatology leading to faster diagnosis. However, the converse is also true; low-grade tumours with favourable characteristics may have slow progression of symptomatology contributing to sustained and progressive acquired disabilities and prolonged TDIs. In the latter group, it can be predicted that reducing the TDI would reduce the acquisition of neurological injury and therefore subsequent disability, the most common acquired disabilities affecting vision, mobility and cognitive capacity. Furthermore, shortening the TDI for this subgroup would increase the likelihood of achieving complete tumour resection and reduce the treatment burden required for a cure, thus limiting the late effects of treatments such as radio- therapy on growth, fertility and cognitive function later in life.

We hypothesise that cases with longer TDIs have different clinicopathological characteristics to those with shorter TDIs. We have studied this using national quality improvement cohort data derived from the HeadSmart service evaluation. The results will inform the ongoing awareness campaign about patient groups at greatest risk of delays.

\section{Methods}

Anonymised patient diagnostic data were collected from 18 treatment centres across the UK for incident cases of brain tumours from January 2011 to May 2013. This was part of a quality improvement project, and a full report including detailed methodology has previously been published. $\underline{8}$

Data including age at diagnosis, date of symptom onset, date of first presentation to healthcare with symptoms attributable to the tumour, date of tumour diagnosis, tumour 
histology and tumour location were collected. Patients aged over 18 at time of diagnosis, patients with a diagnosis of spinal tumours and patient data submitted with two or more key dates missing were excluded from analysis.

The key outcome measures, TDI, patient interval (PI) and system interval (SI), were calculated for each patient. TDI was defined as the interval between date of symptom onset and diagnosis. PI was defined as the interval between symptom onset and initial presentation to a healthcare professional. SI was defined as the interval between initial presentation to healthcare and diagnosis. $\underline{9}$

Subgroup analysis by age at diagnosis, tumour grade and tumour location was also performed.

A total of 778 patient data records were submitted; 68 patients were subsequently excluded for failing one or more of the following checks: over 18 years of age at diagnosis $(n=18)$, spinal tumours $(n=8)$, two key dates missing $(n=19)$, three key dates missing $(n=1)$, date of diagnosis missing $(n=2)$ and illogical date sequence $(n=26)$. Therefore, 710 patients were eligible for analysis.

\section{Results}

\section{Cohort characteristics}

The characteristics of the study population are detailed in table 1. Young children aged under 5 years accounted for $38 \%$ of cases, with a peak age at diagnosis of 2 years (figure 1 ). The most common tumour location was in the posterior fossa $(n=320)$ including the cerebellum $(n=249)$ and brainstem $(n=71)$, followed by the midline supratentorial area $(n=222)$ and cerebral hemispheres $(n=159)$.

Low-grade astrocytoma (grades 1 and 2) was the most common tumour type in all three age groups, constituting $30 \%$ (79/267), 33\% (97/287) and 37\% (58/156) of tumours in the under 5, 5-11 and 12-18 age groups, respectively. Grading was not possible for 61 tumours, including all 35 diffuse intrinsic pontine gliomas as they were diagnosed on imaging alone. The distribution of patients across the age groups differed significantly between tumour locations ( $p=0.003$ ). Patients aged under 5 years and $5-11$ years together comprised the majority of brainstem $(62 / 71 ; 87 \%)$, central $(173 / 222 ; 78 \%)$ and cerebellar tumours (204/249; 82\%). The $12-18$ age groups constituted $13 \%$ (9/71) of brainstem tumours, $22 \%$ $(49 / 222)$ of central tumours, $18 \%$ (45/249) of cerebellar tumours and $33 \%(53 / 159)$ of cerebral hemisphere tumours. The distribution of patients across the age groups also differed with tumour grade $(p=0.047)$. Patients aged $5-11$ years constituted $46 \%(117 / 257)$ of high-grade tumours compared with $37 \%$ (144/392) of low-grade tumours. Patients aged $12-18$ years accounted for $18 \%(46 / 257)$ of high-grade tumours and $24 \%$ (95/392) of low-grade tumours. Tumour grade composition differed significantly between tumour locations $(p=0.003)$ in the 640 cases with complete WHO tumour grade and tumour location data. Low-grade tumours constituted $78 \%(161 / 207)$ of central tumours, compared with52\% (126/243), 53\% (81/154) and 58\% (21/36) of tumours in the cerebellum, cerebral hemisphere and brainstem, respectively.

\section{Interval analysis}

Interval analysis focused on the degree of the skew of the interval data summarised by the 
difference between mean and median values. This difference has been used to rank the interval data in different subgroups.

\section{Tumour location}

From the key outcome measures for tumour location, central tumours had the longest intervals compared with other locations, with a median TDI of 10.5 weeks, median PI of 3.2 weeks and median SI of 2.9 weeks (figure 2). The greatest contribution to TDI came from the system intervals for all tumour types.

\section{Tumour grade}

Low-grade tumours had a significantly higher TDI compared with high-grade tumours; a median of 10.4 weeks in low-grade compared with 6 weeks in high-grade tumours. Lowgrade tumours also showed the most skewed distribution, with a median of 10.4 weeks but a mean TDI of 28.2 weeks, a difference of 17.8 weeks (figure 2). The system interval contributed to the majority of the TDI for low-grade tumours.

\section{Tumour subtype}

Looking at the TDI, ranking of tumour subtypes identified craniopharyngioma, low-grade glioma, optic pathway gliomas and germ cell tumours as highest, with median TDIs of 15.1, $11.9,10.4$ and 5.6 weeks, respectively (figure $3 \mathrm{~A}$ ). Further stratification by tumour location and common subtypes is illustrated in figure 4. Craniopharyngiomas and low-grade glioma had the longest median patient intervals of 4.4 and 4.0 weeks, respectively (figure 3B). The tumour types with shorter TDIs have a less pronounced skew between mean and median and more equal distribution of intervals between patient and system interval.

The tumours with the highest ranked TDIs were midline tumours where the greatest proportion of delay was from the SI rather than the PI. Craniopharyngioma, optic pathway glioma and low-grade glioma had the longest median system intervals of

3.4, 3.3 and 2.9 weeks, respectively (figure $3 \mathrm{C}$ ).

\section{Age}

The 12-18 age group were found to have the longest delay with a median of 12.1 weeks, compared with 6 weeks for the under 5 age group and 8 weeks for the 5-11 age group $(p<0.001)$ (figure 5).

\section{Discussion}

We have studied the impact of clinical factors on diagnostic intervals identified as part of a national quality improvement project seeking to accelerate diagnosis for children with brain tumours across the UK.

These data highlight that anatomical midline tumours, particularly craniopharyngioma, lowgrade gliomas, optic pathway glioma and malignant intracranial germ cell tumours, present 
with the longest TDIs where the SI exceeds the PI, indicating the greatest potential for system intervention. In contrast, the cerebellar and brainstem tumours have the shortest median TDI with the least skewed distribution.

\section{System interval}

A lengthy $\mathrm{SI}$ suggests that patients and their families are attending healthcare services, but the diagnosis of a brain tumour is not always made promptly. We know that childhood brain tumours pose a particular diagnostic challenge as the signs and symptoms that precede diagnosis are non-specific and vary in presentation depending on age, location and tumour grade. A previous systematic review categorised symptoms of brain tumours based on location.10 In terms of anatomical midline tumours, central tumours most commonly present with headache, abnormal eye movements and squint, while supratentorial tumours present with raised intracranial pressure, seizures and papilloedema.10 A lack of awareness among healthcare professionals of the varying presentations as well as the perception of rarity means that a brain tumour is not often considered as a differential.

Along the pathway of initial symptom onset to diagnosis, symptom acquisition linked to tumour growth inevitably occurs. The tumours identified in this study with long TDIs, namely craniopharyngiomas, low-grade gliomas and optic pathway gliomas, tend to be slow growing and so the acquisition of symptoms will also be slow, which can be falsely reassuring to the clinician on initial review. Other contributors to a lengthy SI could be a lack of resources within the healthcare system. Waiting times for secondary care clinics or for an MRI under general anaesthetic can cause substantial delays of weeks or months. The National Institute for Health and Care Excellence (NICE)-accredited RCPCH guideline suggests that a child or young person with a suspected brain tumour should wait no longer than 4 weeks for an MRI. The importance of clear and concise communication of the concern of a brain tumour within referral letters and MRI requests quoting the NICE-accredited HeadSmart guidance should allow prioritisation of the services.

While it is difficult to make change to NHS resources, education to improve awareness among healthcare professionals to aid early diagnosis is imperative. A new decision support tool for each symptom has been developed to allow clinicians to review brief but pertinent checklists derived from the NICE-accredited clinical guideline in order to decide whether to reassure, review or refer (scan) their patient.11 These have been devised for quick and easy use within a 10 min consultation. Anecdotally, children

with the longest delays often pass through a variety of specialties before a diagnosis of a brain tumour is considered. A subspecialty poster, linking symptoms that may present to a certain subspecialty to the location of a potential brain tumour has also been designed to enhance awareness (figure 6). While professional dissemination through national and international conference presentations, national RCGP and RCPCH workshops and free open-access medical education (FOAMed) blogs will have enhanced awareness and confidence among healthcare professionals, a wider reach through accredited e-learning platforms would be beneficial. 


\section{Diagnostic delays in young people}

As in other published studies, we have found that TDIs also increased with age $(<5,5-11$ and 12-18) with the PI contributing the most towards TDI in the 12-18 age group.12 13 Survival rates of cancers in adolescents are comparatively worse than in younger children. $A$ study looking at a series of children and young people with soft-tissue sarcomas showed that a higher mortality rate was associated with a longer symptom interval, and that this interval was longer in adolescents than younger children.14 A further study looking at symptom interval in paediatric patients with solid tumours found that the PI made up twothirds of the symptom interval in adolescents compared with one-third in younger children.15 This delayed presentation trend has been noted across all specialties and as such there has been an increasing focus on teenage and young adolescent (TYA) groups in healthcare over the last decade.16-18 The reasons for delays in the TYA subgroup are multifactorial; adolescence is a period of unique psychological and physiological change. It is not surprising that the greatest contribution to a long TDI in this age group is due to the PI. It is well known that young people have difficulties forming therapeutic relationship with healthcare professionals and accessing health services.19 A lack of awareness of the seriousness of their symptoms or a sense of embarrassment regarding certain symptoms such as delayed puberty can result in avoidance or delay in identifying ways to engage young people and empowering them with knowledge and resources to take ownership of their own health may create an opportunity for earlier diagnosis.

We know that adolescents use the internet frequently to access healthcare information.20 A survey of internet use reported $90 \%$ of adolescents 15-24 years old had accessed the internet and $75 \%$ of these had used the internet for health information.21 The top three subjects searched were specific diseases such as cancer, sexual health and weight loss/gain. Reassuringly, the information they found allowed them to start a conversation with their peers or a healthcare professional.21 These findings formed the basis for revising the HeadSmart campaign in order to target and engage the TYA population.

The campaign re-launched in 2017 after extensive engagement with TYA advisors who designed the new logo and branding. Given that adolescents are more likely to search the internet for healthcare advice, the HeadSmart website was redesigned in consultation with TYA advisors to ensure that it met their needs. Important changes have included an eyecatching symbol-based representation of symptoms, as well as a musical video to highlight all symptoms, especially puberty in an informative, humorous way as a priority.4 Further work including school visits, increasing social media presence and the use of celebrity ambassadors as role models to boost engagement with young people is ongoing.

\section{Strengths and limitations}

A key strength of this study is its population-based characteristics. Compliance with data collection was high and more than $70 \%$ of predicted new diagnoses were captured, allowing a representative national overview of brain tumour diagnosis. While the data show strong patterns with regards to TDI, there are certain limitations to acknowledge. The weaknesses of the data lie in the reliance on recall for historical details, the lack of a control group or a randomised intervention, and the limited dataset for further analysis. As data were collected anonymously via a retrospective audit, longitudinal follow-up is not possible. This 
will be crucial in future work in order to assess any correlation between TDI and long-term disability or survival. Nevertheless, this analysis has highlighted that symptom onset associated with a brain tumour can dramatically pre-date diagnosis by many months for particular tumour types and age groups. The onset of brain tumour symptoms can be assumed to represent the onset of progressive brain injury. If that progression is slow due to a low-grade tumour growth, it offers a longer opportunity for clinical intervention to shorten that delay and therefore reduce the progressive brain injury that can lead to a number of life-changing events. This includes vision loss, motor or sensory deficits, sustained white matter damage due to raised intracranial pressure affecting cognition, as well as irreversible progressive hypothalamic hormone deficiencies.

Modern therapies for brain tumours involve surgery, radiotherapy and in many cases chemotherapy. Each is associated with risks of brain injury which are cumulative. Early diagnosis will allow both a greater chance of curative surgical resection and use of a less aggressive adjuvant therapy plan reducing the side effects and late effects for these children and young people.

\section{Take home messages for clinicians}

These data suggest that the location of the tumour has an effect on the length of time to diagnosis. Brain tumour presentation is unique in that the constellation of symptoms is dependent on the location of the tumour in the brain.10 Being aware of the tumours that present with longer delays will enable us to familiarise ourselves with those symptom clusters in order to diagnose them earlier. Cerebellar symptoms are often quickly identified as a concerning pattern; central tumours are taking longer to diagnose because they present with more subtle, non-specific symptoms (eg, headache and visual symptoms). As central tumours are also often slow-growing, the cognitive bias that a sinister diagnosis would reveal itself quickly can falsely reassure both the clinician and the family. Here are some general tips to use to ensure the diagnosis is not missed:

Always include a brain tumour on your differential diagnosis list when faced with persistent non-specific symptoms.

Use the evidence-based HeadSmart symptom list4 as a checklist within your consultation to help identify which symptoms are present and for how long.

Monitor this checklist on review to identify evolving symptom patterns as there is an accumulation and acquisition of symptoms as the tumour grows.

\section{Conclusion}

Given that more than $70 \%$ of children and young people now survive a brain tumour diagnosis, this work would suggest there is a great opportunity to reduce the overall burden of disability. Raising awareness among both healthcare professionals and the public through targeted educative strategies will ensure the earliest possible diagnosis in order to limit pre-diagnostic brain injury for these patients. 
Acknowledgements The HeadSmart campaign is a partnership between the Children's Brain Tumour Research Centre at the University of Nottingham, the Brain Tumour Charity and the Royal College of Paediatrics and Child Health. The authors would like to thank The Health Foundation for providing the initial funding to set up the campaign, the professional colleges that endorsed the RCPCH guidelines and the HeadSmart campaign, the Children's Cancer and Leukaemia Group, and all clinical and community champions for their support to the HeadSmart project.

Collaborators On behalf of the HeadSmart: Early diagnosis of brain tumours campaign group: Veronica Neefjes, Royal Aberdeen Children's Hospital; Anthony McCarthy, Royal Belfast Hospital for Sick Children; Martin English, Birmingham Children's Hospital; Steve Lowis, Rachel Perrow, Bristol Royal Hospital for Children; Matthew Garnett, Addenbrooke's Hospital; Cathy Morley Jacob, Heidi Traunecker, Children's Hospital for Wales; Jane Pears, Our Lady's Children's Hospital; Alf Nicholson, Temple Street Children's University Hospital; Hamish Wallace, Mark Brougham, Royal Hospital for Sick Children, Edinburgh; Jairam Sastry, Royal Hospital for Sick Children, Glasgow; Antony Michalski, Great Ormond Street Hospital; Simone Wilkins, Leeds Children's Hospital; Barry Pizer, James Hayden, Alder Hey Children's Hospital; Eddy Estlin, Royal Manchester Children's Hospital; Juliet Hale, The Great North Children's Hospital; Sophie Wilne, Nottingham Children's Hospital; Denise Tritton, John Radcliffe Hospital; Darren Hargrave, The Royal Marsden Hospital; Vicki Lee, Sheffield Children's Hospital.

Contributors DW, SW and J-FL designed the study. LM, J-FL and DS analysed the data. DS drafted the manuscript which was reviewed and edited by DW, SW and J-FL.

The HeadSmart Clinical Champions submitted data from their centres for the study.

Funding The authors have not declared a specific grant for this research from any funding agency in the public, commercial or not-for-profit sectors.

Competing interests None declared.

Patient consent for publication Not required.

Provenance and peer review Not commissioned; externally peer reviewed.

Data availability statement No data are available. 


\section{References}

1 Cancer Research UK Statistics. Available: http://www. cancerresearchuk. org/ healthprofessional/ cancer- statistics/ childrens- cancers\# heading-Zero

2 Hjern A, Lindblad F, Boman KK. Disability in adult survivors of childhood cancer: a Swedish national cohort study. J Clin Oncol 2007;25:5262-6.

3 Wilne S, Koller K, Collier J, et al. The diagnosis of brain tumours in children: a guideline to assist healthcare professionals in the assessment of children who may have a brain tumour. Arch Dis Child 2010;95:534-9.

4 The HeadSmart. Available: www. headsmart. org. uk

5 HeadSmart Be Brain Tumour Aware. A new clinical guideline from the Royal College of Paediatrics and Child Health with a national awareness campaign accelerates brain tumor diagnosis in UK children- "HeadSmart: Be Brain Tumour Aware". Neuro Oncol 2016;18:44554.

6 Cancer Research UK. Achieving world class cancer outcomes: a strategy for England 20152020. Report of independent Cancer Taskforce. Available: http://www.cancerresearchuk. org/ sites/ default/ files/ achieving_world- class_cancer_outcomes_-_a_strategy_for_ england_2015-2020. pdf

7 Kukal K, Dobrovoljac M, Boltshauser E, et al. Does diagnostic delay result in decreased survival in paediatric brain tumours? Eur J Pediatr 2009;168:303-10.

8 HeadSmart: Be Brain Tumour Aware. A new clinical guideline from the Royal College of Paediatrics and Child Health with a national awareness campaign accelerates brain tumor diagnosis in UK children-"HeadSmart: Be Brain Tumour Aware". Neuro Oncol 2016;18:44554.

9 Weller D, Vedsted P, Rubin G, et al. The Aarhus statement: improving design and reporting of studies on early cancer diagnosis. Br J Cancer 2012;106:1262-7.

10 Wilne S, Collier J, Kennedy C, et al. Presentation of childhood CNS tumours: a systematic review and meta-analysis. Lancet Oncol 2007;8:685-95.

11 HeadSmart decision support tools. Available: https://www. headsmart. org. uk/ clinical/decision- support- tool/

12 Haimi MM, Nahum MP, Arush MWB. Delay in diagnosis of children with cancer: a retrospective study of 315 children. Pediatr Hematol Oncol 2004;21:37-48.

13 Dang-Tan T, Trottier H, Mery LS, et al. Delays in diagnosis and treatment among children and adolescents with cancer in Canada. Pediatr Blood Cancer 2008;51:468-74.

14 Ferrari $A$, Miceli $R$, Casanova $M$, et al. The symptom interval in children and adolescents with soft tissue sarcomas. Cancer 2010;116:177-83.

15 Veneroni L, Mariani L, Lo Vullo S, et al. Symptom interval in pediatric patients solid tumors: adolescents are at greater risk of late diagnosis. Pediatr Blood Cancer 2013;60:60510.

16 Kennedy I. Getting it right for children and young people. Overcoming cultural barriers in the NHS so as to meet their need. London, UK: Department of Health, 2010. http://www. dh. gov. uk

$17 \mathrm{RCPCH}$. Not just a phase-a guide to the participation of children and young people in health services, 2010.

18 Wilkinson D, Robinson AJ. You're Welcome quality criteria: making health services youngpeople-friendly. Sex Transm Infect 2009;85:233-4.

19 Jacobson L, Richardson G, Parry-Langdon N, et al. How do teenagers and primary healthcare providers view each other? An overview of key themes. Br J Gen Pract 
2001;51:811-6.

20 Gray NJ, Klein JD, Noyce PR, et al. Health information-seeking behaviour in adolescence: the place of the internet. Soc Sci Med 2005;60:1467-78.

21 Rideout V. Generation Rx. com: how young people use the internet for health information. Menlo Park CA: Kaiser Family foundation, 2001. 